\title{
Axisymmetric Torsion of an Elastic Layer Sandwiched between Two Elastic Half-Spaces with Two Interfaced Cracks
}

https://doi.org/10.2478/sgem-2019-0006

received October 10, 2018; accepted January 15, 2019.

\begin{abstract}
The present article examines the problem related to the axisymmetric torsion of an elastic layer by a circular rigid disc at the symmetry plane. The layer is sandwiched between two similar elastic half-spaces with two penny-shaped cracks symmetrically located at the interfaces between the two bonded dissimilar media. The mixed boundary-value problem is transformed, by means of the Hankel integral transformation, to dual integral equations, that are reduced, to a Fredholm integral equation of the second kind. The numerical methods are used to convert the resulting system to a system of infinite algebraic equations. Some physical quantities such as the stress intensity factor and the moment are calculated and presented numerically according to some relevant parameters. The numerical results show that the discontinuities around the crack and the inclusion cause a large increase in the stresses that decay with distance from the disc-loaded. Furthermore, the dependence of the stress intensity factor on the disc size, the distance between the crack and the disc, and the shear parameter is also observerd.
\end{abstract}

Keywords: Axisymmetric torsion; Penny-shaped crack; Dual integral equations; Fredholm integral equations.

*Corresponding author: Fateh Madani, Mechanical Engineering and Development Laboratory, Mechanical Engineering Department, National Polytechnic School, 10, Avenue Hassen Badi- B.P. 182-16200, ElHarrach, Algiers, Algeria, E-mail: madanifateh1984@yahoo.com Belkacem Kebli: Mechanical Engineering and Development Laboratory, Mechanical Engineering Department, National Polytechnic School, 10, Avenue Hassen Badi- B.P. 182- 16200, El-Harrach, Algiers, Algeria

\section{Introduction}

The class of problems related to the behavior of rigid disc inclusions embedded in bonded contact with an elastic medium, has been a subject of much interest in geomechanics, civil engineering, and applied mechanics. This work is motivated by both theoretical and practical interests in the problems of turbines disks, some pipes, and many industrial applications. It may give a better understanding of the behavior of foundations under external loads. In structure-medium interaction problems arising in foundation engineering, the foundation is usually modeled using a rigid or flexible inclusion having circular, strip, rectangular, or arbitrary shape. Nowadays, composites play a very important role in geomechanics engineering.

It is common knowledge that all existing structural materials contain different inter- and intra-component defects (cracks, delaminations, etc.) [1]. The problem of the torsion of an infinite elastic medium by a rigid inclusion (deeply embedded) was considered by Selvadurai $[2,3]$. His results depend on the rotational and translational stiffnesses of the embedded rigid circulardisc. The problem of the torsion of an elastic half- space was considered, at first, by Reissner and Sagoci [4]. They studied the static interaction of a rigid disc and an elastic isotropic halfspace for which they obtained the solution by means of the spheroidal coordinates. The same problem was solved by Sneddon [5] using a different method. He used the Hankel transforms method for reducing the problem to a pair of dual integral equations. Collins [6] treated the torsional problem of an elastic half-space by assuming the displacement at any point in the half- space to be due to a distribution of wave sources over the part of the free surface in contact with the disc. The solution of the forced vibration problem of elastic layer of finite thickness when the lower face is either stress free or rigidly clamped was given by Gladwell [7]. Pak and Saphores [8] provided an analytical formulation for the general torsional problem 
of a rigid disc embedded in an isotropic half-space. Besides, Bacci and Bennati [9] considered the torsional of circular rigid disc adhered to the upper surface of an elastic layer fixed to an undefonnable support. More recently, Singh et al. [10] studied the torsional of a nonhomogeneous, isotropic, half- space by rotating a circular part of its boundary surface. Cai and Zue [11] discussed the torsional vibration of a rigid disc bonded to a poroelastic multilayered medium.. Yu [12] studied the forced torsional oscillations inside the multilayered solid. The elastodynamic Green's function of the center of rotation and a point load method were used to solve the problem. Pal and Mandal [13] considered the forced torsional oscillations of a transversely isotropic elastic half- space under the action of an inside rigid disc. A similar problem with the rocking rotation was solved later on by Ahmadi and Eskandari [14]. They used an appropriate Green's function to write the mixed boundary-value problem posed as a dual integral equation. All these problems are based on the theory of Hankel integral transformation in order to bring the mixed boundary -value problem into a system of dual integral equations. Then, the corresponding solution is sought from an integral equation of Fredholm type.

The torsional of elastic layers with a penny -shaped crack was considered by some researchers. Sih and Chen [15] studied the problem of a penny-shaped crack in layered composite under a uniform torsional stress. The displacement and stress fields throughout the composite were obtained by solving a standard Fredholm integral equation of the second kind. Low [16] investigated a problem of the effects of embedded flaws in the form of an inclusion or a crack in an elastic half- space subjected to torsional deformations. The corresponding Fredholm integral equations were solved numerically by quadrature approach. The same method was used by Dhawan [17] for solving the problem of a rigid disc attached to an elastic half-space with an internal crack. By using Hankel and Laplace transforms and taking numerical inversion of Laplace transform, Basu and Mandal [18] treated the torsional load on a penny-shaped crack in an elastic layer sandwiched between two elastic half-spaces. The purpose of this article is to study an axisymmetric torsion of an embedded circular rigid disc in bonded contact with an isotropic elastic layer sandwiched between two elastic half-spaces with two penny-shaped cracks symmetrically located at each of the two interfaces between the layer and the half-spaces. A similar method was used in arecently published work by Madani and Kebli [20], dealing with the case of a penny-shaped crack problem in the interior of a homogeneous elastic material at the symmetry plane, under an axisymmetric torsion by two circular rigid discs symmetrically located in the elastic medium.

\section{Basic Equations of the Problem}

In view of the axial symmetry of the problem, it is natural to consider $(r, \theta, z)$ the cylindrical polar co-ordinates. Here we consider an axisymmetric torsion of an embedded circular rigid disc with a radius $b$ at the symmetry plane $z=0$ in an isotropic and homogeneous layer of thickness $2 h$ and with one material sandwiched between two halfspaces of the second material. Two penny -shaped cracks with a radius $a$ were symmetrically located at the interface between the layer and the half- spaces $z= \pm h$. The faces of the cracks are assumed to be stress free while the discs rotate with an equal angle $\omega$ about the $z$-axis passing through their center as shown in Figure .1 .

Owing to symmetry about the $z=0$ plane, it is sufficient to consider the problem in the upper half- space where $z$ $\geq 0$. In this case of an axisymmetric torsion problem, the displacement vector assumes the form $\left(0, u_{\theta}, 0\right)$ in the cylindrical polar coordinate system $(r, \theta, z)$. It is convenient to identify a layer region (superscript (1)) occupying the region $r \in(0, \infty) ; z \in\left(0, h^{-}\right)$and a layer region (superscript (2)) occupying the region $r \in(0, \infty) ; z \in\left(h^{-}, \infty\right)$. The signs $h^{+}$and $h^{-}$" denote the variables in the upper surface and the lower surface of plane $z=h$, respectively.

The only non-zero components of stress are given by

$$
\tau^{i}{ }_{\theta z}=G_{i} \frac{\partial u_{\theta}^{i}}{\partial z}, \quad \tau_{\theta r}^{i}=G_{i} r \frac{\partial}{\partial r}\left(\frac{u_{\theta}^{i}}{r}\right), \quad i=1,2
$$

where $u_{\theta}=u_{\theta}(r, z)$ and $G i$ is the shear modulus of the material. As the torsion of the homogeneous material is static, the displacement $u_{\theta}(r, z)$ must satisfy

$$
\frac{\partial^{2} u_{\theta}}{\partial r^{2}}+\frac{\partial u_{\theta}}{r \partial r}-\frac{u_{\theta}}{r^{2}}+\frac{\partial^{2} u_{\theta}}{\partial z^{2}}=0
$$

By means of Hankel's transformation, integral and its inverse given in [19],

$$
F(\lambda, z)=\int_{0}^{\infty} f(r, z) r J_{1}(\lambda r) d r
$$

And

$$
f(r, z)=\int_{0}^{\infty} F(\lambda, z) \lambda J_{1}(\lambda r) d \lambda
$$




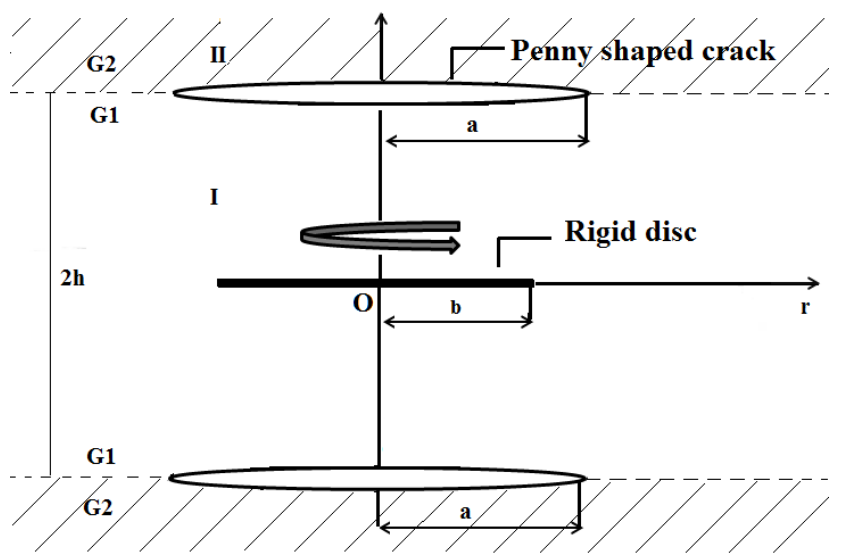

Figure 1: Geometry and coordinate system.

The solution of equation (2) for the regions $I(0 \leq z \leq h)$ and $I I(z \geq h)$ is expressed as

$$
u_{\theta}^{(i)}(r, z)=\int_{0}^{\infty}\left[A_{i}(\lambda) e^{-\lambda z}+B_{i}(\lambda) e^{\lambda z}\right] J_{1}(\lambda r) d \lambda i=, 2
$$

where $\lambda$ is the transform variable, $J 1$ is the Bessel function of the first kind of order one, and $A i$ and $B i$ are unknown functions

\section{Boundary and Continuity Conditions}

We consider the regularity conditions at infinity, the symmetry plane condition at $z=0$, and the boundary and continuity conditions at the bonded interfaces $z=h^{+}$and $h^{-}$. Therefore, we find the following conditions

$$
\begin{array}{ccc}
\lim _{r, z \rightarrow \infty} u_{\theta}^{i}(r, z)=0, \quad & \lim _{r, z \rightarrow \infty} \tau_{\theta z}^{i}(r, z)=0 \\
\tau_{\theta z}{ }^{(1)}(r, 0)=0, & r>b & \\
\tau_{\theta z}^{(1)}\left(r, h^{-}\right)-\tau_{\theta z}{ }^{(2)}\left(r, h^{+}\right)=0, & r \geq a \\
u_{\theta}^{(1)}\left(r, h^{-}\right)-u_{\theta}^{(2)}\left(r, h^{+}\right)=0, & r \geq a \\
\tau_{\theta z}^{(2)}\left(r, h^{+}\right)=\tau_{\theta z}{ }^{(1)}\left(r, h^{-}\right)=0 & r<a \\
u_{\theta}{ }^{(1)}(r, 0)=\omega r & r \leq b
\end{array}
$$

Applying the regularity conditions at infinity given in Eq. (4a), we obtain

$$
\begin{gathered}
u_{\theta}^{(1)}(r, z)=\int_{0}^{\infty}\left[A_{1}(\lambda) e^{-\lambda z}+B_{1}(\lambda) e^{\lambda z}\right] J_{1}(\lambda r) d \lambda \\
\tau_{\theta z}{ }^{(1)}(r, z)=G_{1} \int_{0}^{\infty} \lambda\left[-A_{1}(\lambda) e^{-\lambda z}+B_{1}(\lambda) e^{\lambda z}\right] J_{1}(\lambda r) d \lambda \\
u_{\theta}{ }^{(2)}(r, z)=\int_{0}^{\infty}\left[A_{2}(\lambda) e^{-\lambda z}\right] J_{1}(\lambda r) d \lambda \\
\tau_{\theta z}{ }^{(2)}(r, z)=-G_{2} \int_{0}^{\infty} \lambda\left[-A_{2}(\lambda) e^{-\lambda z}\right] J_{1}(\lambda r) d \lambda
\end{gathered}
$$

where $A_{1}(\lambda), B_{1}(\lambda)$, and $A_{2}(\lambda)$ are arbitrary functions that need to be determined by satisfying the boundary and continuity conditions.

The boundary and the continuity conditions in Eqs. (4c) and (4e) lead to

$$
\tau_{\theta z}^{(2)}\left(r, h^{+}\right)-\tau_{\theta z}{ }^{(1)}\left(r, h^{-}\right)=0
$$

The above equation implies

$$
A_{2}(\lambda)=\gamma\left(A_{1}(\lambda)-B_{1}(\lambda) \mathrm{e}^{2 \lambda h}\right)
$$

where $\gamma=\frac{G_{1}}{G_{2}}$.

The mixed boundary conditions Eqs. (4e), (4d), (4f), and (4b) are satisfied if $A_{1}$ and $B_{1}$ are solutions of the following dual integral equations:

$$
\int_{0}^{\infty} \lambda\left[B_{1}(\lambda) e^{\lambda h}-A_{1}(\lambda) e^{-\lambda h}\right] J_{1}(\lambda r) d \lambda=0 \quad 0 \leq r<a
$$

$$
\begin{array}{cr}
\int_{0}^{\infty}\left[(1-\gamma) e^{-\lambda h} A_{1}(\lambda)+(1+\gamma) e^{\lambda h} B_{1}(\lambda)\right] J_{1}(\lambda r) d \lambda=0 & r \geq a \\
\int_{0}^{\infty}\left[A_{1}(\lambda)+B_{1}(\lambda)\right] J_{1}(\lambda r) d \lambda=\omega r & 0 \leq r \leq b \\
\int_{0}^{\infty} \lambda\left[B_{1}(\lambda)-A_{1}(\lambda)\right] J_{1}(\lambda r) d \lambda=0 & r>b
\end{array}
$$

\subsection{Limiting Cases}

Let's take the limit $a \rightarrow 0$ and the nonhomogeneity parameter $y=1$, one can obtain the closed-form solution pertinent to the torsional rotation of a rigid disc embedded in a homogeneous elastic full-space. Owing to the symmetry of the full-space case with respect to the plane of the disc, it can be deduced that $\tau_{\theta z}$ is zero for $r>a$ at the disc plane. This situation corresponds exactly to the torsion of a homogeneous elastic half-space by a circular rigid disc $(0<r<a, z=0)$ bonded to the surface. This is adapted to the problem concerning isotropic half-space considered by Reissner and Sagoci [4]. 
By taking the parameter $y=1$, the problem is simplified to the torsional rotation of a rigid circular inclusion attached to an elastic half-space with internal crack and the dual integral equations become

$$
\begin{array}{rrr}
\int_{0}^{\infty} \lambda\left[B_{1}(\lambda) e^{\lambda h}-A_{1}(\lambda) e^{-\lambda h}\right] J_{1}(\lambda r) d \lambda=0 & 0 \leq r<a(9 \mathrm{a}) \\
\int_{0}^{\infty}\left[2 e^{\lambda h} B_{1}(\lambda)\right] J_{1}(\lambda r) d \lambda=0 & r \geq a \quad \text { (9b) } \\
\int_{0}^{\infty}\left[A_{1}(\lambda)+B_{1}(\lambda)\right] J_{1}(\lambda r) d \lambda=\omega r & 0 \leq r \leq b & \text { (9c) } \\
\int_{0}^{\infty} \lambda\left[B_{1}(\lambda)-A_{1}(\lambda)\right] J_{1}(\lambda r) d \lambda=0 & r>b \quad(9 \mathrm{~d})
\end{array}
$$

This system of dual integral equations has the same meaning as Eqs. (13), (14), (15), and (16) in Dhawan's paper [17].

\section{Reduction of the dual integral equations}

Equations $8 \mathrm{~b}$ and $8 \mathrm{~d}$ are identically satisfied if we introduce the following representation:

$$
\begin{gathered}
{\left[(1-\gamma) e^{-\lambda h} A_{1}(\lambda)+(1+\gamma) e^{\lambda h} B_{1}(\lambda)=\sqrt{\lambda} \int_{0}^{a} \sqrt{t} \phi(t)\right]_{\frac{3}{2}}(\lambda t) d t} \\
B_{1}(\lambda)-A_{1}(\lambda)=\sqrt{\lambda} \int_{0}^{b} \sqrt{t} \psi(t) J_{\frac{1}{2}}(\lambda t) d t
\end{gathered}
$$

where $J_{\frac{1}{2}}$ and $J_{\frac{3}{2}}$ are the Bessel functions of the first kind of order $\frac{1}{2}$ and $\frac{3}{2}$, respectively. The unknown functions are given by the following equation:

$$
\begin{aligned}
& A_{1}(\lambda)=\frac{e^{-\lambda h}}{p(\lambda)(1+\gamma)} \sqrt{\lambda} \int_{0}^{a} \sqrt{t} \phi(t) J_{\frac{3}{2}}(\lambda t) d t-\frac{\sqrt{\lambda}}{p(\lambda)} \int_{0}^{b} \sqrt{t} \psi(t) J_{\frac{1}{2}}(\lambda t) d t \\
& B_{1}(\lambda)=\frac{e^{-\lambda h}}{p(\lambda)(1+\gamma)} \sqrt{\lambda} \int_{0}^{a} \sqrt{t} \phi(t) J_{\frac{3}{2}}(\lambda t) d t+\sqrt{\lambda}\left(1-\frac{\sqrt{\lambda}}{p(\lambda)} \int_{0}^{b} \sqrt{t} \psi(t)\right)_{\frac{1}{2}}(\lambda t) d t
\end{aligned}
$$

where $p(\lambda)=\left(\frac{1-\gamma}{1+\gamma}\right) e^{-2 \lambda h_{+}}$and $\phi(t)$ and $\psi(t)$ are continuous unknown functions of $t$ defined over two intervals $0 \leq t<a$ and $0 \leq t<b$, respectively. Substituting $A 1(\lambda)$ and $B 1(\lambda)$ in Eqs. (8a) and (8c), we get

$$
\begin{gathered}
\left.\left.\int_{0}^{a} \sqrt{t} \varphi(t) d t \int_{0}^{\infty} \lambda_{2}^{\frac{3}{2}} f_{11}(\lambda)\right]_{\frac{3}{2}}(\lambda t) J_{1}(\lambda r) d \lambda+\int_{0}^{b} \sqrt{t} \psi(t) d t \int_{0}^{\infty} \lambda^{\frac{3}{2}} f_{12}(\lambda)\right]_{\frac{1}{2}}(\lambda t) J_{1}(\lambda r) d \lambda \\
=0 r<a \\
\left.\int_{0}^{a} \sqrt{t} \varphi(t) d t \int_{0}^{\infty} \sqrt{\lambda} f_{21}(\lambda) J_{\frac{3}{2}}(\lambda t) J_{1}(\lambda r) d \lambda+\int_{0}^{b} \sqrt{t} \psi(t) d t \int_{0}^{\infty} \sqrt{\lambda} f_{22}(\lambda)\right]_{\frac{1}{2}}(\lambda t) J_{1}(\lambda r) d \lambda \\
=\omega r r<b
\end{gathered}
$$

where

$$
\begin{gathered}
f_{11}(\lambda)=\frac{1}{p(\lambda)}\left(1-e^{-2 \lambda h}\right) \\
f_{12}(\lambda)=(1+\gamma)\left(\left(1-\frac{1}{p(\lambda)}\right) e^{\lambda h}+\frac{1}{p(\lambda)} e^{-\lambda h}\right) \\
f_{21}(\lambda)=\frac{2 e^{-\lambda h}}{p(\lambda)(1+\gamma)} \\
f_{22}(\lambda)=\left(1-\frac{2}{p(\lambda)}\right)
\end{gathered}
$$

The expression for $p(\lambda)$ approaches to 1 for large values of $\lambda$. Equation 14 can be converted to the Abel integral equation by means of the relation $\lambda J_{1}(\lambda r)=\frac{1}{r^{2}} \frac{d}{d r}\left[r^{2} J_{2}(\lambda r)\right]$, and then, by taking into account the integral formula

$$
\int_{0}^{\infty} \sqrt{\lambda} J_{\frac{3}{2}}(\lambda t) J_{2}(\lambda r) d \lambda= \begin{cases}\sqrt{\frac{2}{\pi} \frac{t^{\frac{3}{2}}}{r^{2} \sqrt{\left(r^{2}-t^{2}\right)}}} & t<r \\ 0 & t>r\end{cases}
$$

we obtain the Abel equation corresponding to Eq. (14)

$$
\begin{gathered}
\sqrt{\frac{2}{\pi}} \int_{0}^{r} \frac{t^{2} \varphi(t)}{\sqrt{\left(r^{2}-t^{2}\right)}} d t+r^{2} \int_{0}^{a} \sqrt{t} \varphi(t) d t \int_{0}^{\infty} \lambda^{\frac{1}{2}}\left(f_{11}(\lambda)-1\right) J_{\frac{3}{2}}(\lambda t) J_{2}(\lambda r) d \lambda \\
\quad+r^{2} \int_{0}^{b} \sqrt{t} \psi(t) d t \int_{0}^{\infty} \lambda^{\frac{1}{2}} f_{12}(\lambda) J_{\frac{1}{2}}(\lambda t) J_{2}(\lambda r) d \lambda=0, \quad r<a
\end{gathered}
$$

Next, we invert the last equation by applying the Abel transform formula

$$
\int_{0}^{r} \frac{f(t)}{\sqrt{\left(r^{2}-t^{2}\right)}} d t=g(r) \text { then } f(t)=\frac{2}{\pi} \frac{d}{d t} \int_{0}^{t} \frac{r g(r)}{\sqrt{\left(t^{2}-r^{2}\right)}} d r-
$$

to obtain

$$
\begin{aligned}
& t^{2} \varphi(t)=\sqrt{\frac{2}{\pi}} \frac{d}{d t} \int_{0}^{t} \frac{r^{3}}{\sqrt{\left(t^{2}-r^{2}\right)}}\left[-\int_{0}^{a} \sqrt{\delta} \varphi(\delta) d \delta \int_{0}^{\infty} \lambda^{\frac{1}{2}}\left(f_{11}(\lambda)-1\right)\right]_{\frac{3}{2}}(\lambda \delta) J_{2}(\lambda r) d \lambda \\
&\left.\left.\quad-\int_{0}^{b} \sqrt{\delta} \psi(\delta) d \delta \int_{0}^{\infty} \lambda^{\frac{1}{2}} f_{12}(\lambda)\right]_{\frac{1}{2}}(\lambda t) J_{2}(\lambda r) d \lambda\right] d r=0, \quad r<a
\end{aligned}
$$

For the left-hand side of the above equation, the integral is further simplified by using the following relationship:

$$
\sqrt{\frac{2}{\pi}} \frac{d}{d t} \int_{0}^{t} \frac{r^{3}}{\sqrt{t^{2}-r^{2}}} J_{2}(\lambda r) d r=\sqrt{\lambda} t^{\frac{5}{2}} J_{\frac{3}{2}}(\lambda t)
$$

we obtain the first Fredholm integral equation of the second kind 
$\varphi(t)+\sqrt{t} \int_{0}^{a} \sqrt{\delta} \varphi(\delta) K(t, \delta) d \delta+\sqrt{t} \int_{0}^{b} \sqrt{\delta} \psi(\delta) L(t, \delta) d \delta=0$,

$$
0<t<a
$$

where

$$
\begin{gathered}
K(t, \delta)=\int_{0}^{\infty} \lambda\left(f_{11}(\lambda)-1\right) J_{\frac{3}{2}}(\lambda t) J_{\frac{3}{2}}(\lambda \delta) d \lambda \\
L(t, \delta)=\int_{0}^{\infty} \lambda f_{12}(\lambda) J_{\frac{3}{2}}(\lambda t) J_{\frac{1}{2}}(\lambda \delta) d \lambda
\end{gathered}
$$

Following the similar procedure as before, Eq. (15) is reduced to the second Fredholm integral equation.

Using the following formula

$$
\int_{0}^{\infty} \sqrt{\lambda} J_{\frac{1}{2}}(\lambda t) J_{1}(\lambda r) d \lambda= \begin{cases}\sqrt{\frac{2 t}{\pi}} \frac{1}{r \sqrt{\left(r^{2}-t^{2}\right)}} & t<r \\ 0 & t>r\end{cases}
$$

we obtain the following Abel-type equation:

$$
\begin{aligned}
\frac{1}{r} \sqrt{\frac{2}{\pi}} \int_{0}^{r} \frac{t \psi(t)}{\sqrt{\left(r^{2}-t^{2}\right)}} d t+\int_{0}^{a} \sqrt{t} \varphi(t) d t \int_{0}^{\infty} \sqrt{\lambda} f_{21}(\lambda) J_{\frac{3}{2}}(\lambda t) J_{1}(\lambda r) d \lambda \\
\quad+\int_{0}^{b} \sqrt{t} \psi(t) d t \int_{0}^{\infty} \sqrt{\lambda}\left(f_{22}(\lambda)-1\right)(\lambda) J_{\frac{1}{2}}(\lambda t) J_{1}(\lambda r) d \lambda=\omega r \\
\quad r<b
\end{aligned}
$$

Now, we invert the above equation by applying the Abel transform formula to get

$$
\begin{aligned}
t \psi(t)= & \sqrt{\frac{2}{\pi}} \frac{d}{d t} \int_{0}^{t} \frac{r^{2}}{\sqrt{\left(t^{2}-r^{2}\right)}}\left[\omega r-\int_{0}^{a} \sqrt{\delta} \varphi(\delta) d \delta \int_{0}^{\infty} \sqrt{\lambda} f_{21}(\lambda) J_{\frac{3}{2}}(\lambda \delta) J_{1}(\lambda r) d \lambda\right. \\
& \left.-\int_{0}^{b} \sqrt{\delta} \psi(\delta) d \delta \int_{0}^{\infty} \sqrt{\lambda}\left(f_{22}(\lambda)-1\right) J_{\frac{1}{2}}(\lambda \delta) J_{1}(\lambda r) d \lambda\right] d r, r<b
\end{aligned}
$$

Using the following relationships

$$
\begin{gathered}
\frac{d}{d t} \int_{0}^{t} \frac{r^{3}}{\sqrt{t^{2}-r^{2}}} d r=2 t^{2} \\
\sqrt{\frac{2}{\pi}} \frac{d}{d t} \int_{0}^{t} \frac{r^{2} J_{1}(\lambda r)}{\sqrt{t^{2}-r^{2}}} d r=t \sqrt{\lambda} t J_{\frac{1}{2}}(\lambda t)
\end{gathered}
$$

we get the second Fredholm integral equation of the second kind

$$
\begin{gathered}
\psi(t)+\sqrt{t} \int_{0}^{a} \sqrt{\delta} \varphi(\delta) M(t, \delta) d \delta+\sqrt{t} \int_{0}^{b} \sqrt{\delta} \psi(\delta) N(t, \delta) d \delta=\frac{4 \omega}{\sqrt{2 \pi}} t, \\
0<t<b
\end{gathered}
$$

with the kernel

$$
M(t, \delta)=\int_{0}^{\infty} \lambda f_{21}(\lambda) J_{\frac{1}{2}}(\lambda t) J_{\frac{3}{2}}(\lambda \delta) d \lambda
$$

$$
N(t, \delta)=\int_{0}^{\infty} \lambda\left(f_{22}(\lambda)-1\right) J_{\frac{1}{2}}(\lambda t) J_{\frac{1}{2}}(\lambda \delta) d \lambda
$$

To get a non-dimensionalized equation, from Eqs. (18) to (22), let us change the variables as follows

$$
\delta=\left\{\begin{array}{ll}
a s & 0<\delta<a \\
b s & 0<\delta<b
\end{array}, \quad t= \begin{cases}a u & 0<t<a \\
b u & 0<t<b,\end{cases}\right.
$$

Next, we multiply the above two equations of the system by $\frac{\sqrt{2 \pi}}{4 a \omega} \varphi(a u)$ and $\frac{\sqrt{2 \pi}}{4 b \omega} \psi(b u)$, respectively, and using the following substitutions

$$
\left\{\begin{array}{cl}
\Phi(u)=\frac{\sqrt{2 \pi}}{4 a \omega} \varphi(a u) & \Psi(u)=\frac{\sqrt{2 \pi}}{4 b \omega} \psi(b u) \\
c=\frac{a}{b} \quad \lambda=\frac{x}{b} & H=\frac{h}{b}
\end{array}\right.
$$

we obtain the following equations:

$$
\begin{gathered}
\Phi(u)+c^{2} \sqrt{u} \int_{0}^{1} \sqrt{s} \Phi(s) K(u, s) d s+\frac{1}{\sqrt{c}} \sqrt{u} \int_{0}^{b} \sqrt{s} \Psi(s) L(u, s) d s=0, \quad u<1(25 \mathrm{a}) \\
\Psi(u)+c^{2} \sqrt{c} \sqrt{u} \int_{0}^{1} \sqrt{s} \Phi(s) M(u, s) d s+\sqrt{u} \int_{0}^{b} \sqrt{s} \Psi(s) N(u, s) d s=0, u<1(25 \mathrm{~b})
\end{gathered}
$$

where

$$
\begin{gathered}
K(u, s)=\int_{0}^{\infty} x\left(f_{11}(x)-1\right) J_{\frac{3}{2}}(x c u) J_{\frac{3}{2}}(x c s) d x \\
L(t, \delta)=\int_{0}^{\infty} x f_{12}(x) J_{\frac{3}{2}}(x c u) J_{\frac{1}{2}}(x s) d x \\
M(t, \delta)=\int_{0}^{\infty} x f_{21}(x) J_{\frac{1}{2}}(x u) J_{\frac{3}{2}}(x c s) d x \\
N(t, \delta)=\int_{0}^{\infty} x\left(f_{22}(x)-1\right) J_{\frac{1}{2}}(x u) J_{\frac{1}{2}}(x s) d x
\end{gathered}
$$

\section{Numerical Results and Discussion}

As the kernels $K, L, M$, and $N$ are continuous on the interval $[0,1]$, the system of Fredholm integral equations can be solved by direct or iterative techniques [21]. The midpoint quadrature [22] is used to find the numerical solution for the system given by Eqs. (25a) and (25b). By dividing the interval $[0,1]$ into $\mathrm{N}$ equal subintervals, so that the midpoints are $u=u_{m}=\frac{2 m-1}{2} \quad s=u_{n}=\frac{2 n-1}{2} \quad m, n=1,2 \ldots N$, and by introducing the following notations

$$
\Phi\left(u_{m}\right)=\Phi_{m} \quad \Psi\left(u_{m}\right)=\Psi_{m}
$$




$$
K\left(u_{m}, u_{n}\right)=K_{m n} \quad L\left(u_{m}, u_{n}\right)=L_{m n}
$$

We evaluate numerically the infinite integral $K, L, M$, and $N$ using the Simpson rule. After solving the above system, the unknown coefficients can be obtained.

\subsection{Stress intensity factors}

The stress intensity factors at the edge of the crack and at the rim of the disc are defined, respectively, by the following equations:

$$
\begin{aligned}
& K_{I I I}^{a}=\left.\lim _{r \rightarrow a^{+}} \sqrt{2 \pi(r-a)} \tau_{z \theta}^{1}(r, z)\right|_{z=H} \\
& K_{I I I}^{b}=\left.\lim _{r \rightarrow b^{-}} \sqrt{2 \pi(r-b)} \tau_{z \theta}^{1}(r, z)\right|_{z=0}
\end{aligned}
$$

On the planes $z=h$ for $r \geq a$ and $z=0$, the expressions of stress are given by

$$
\begin{gathered}
\tau_{z \theta}^{1}(r, 0)=G_{1} \int_{0}^{\infty} \lambda^{\frac{3}{2}}\left[\frac{1-e^{-2 \lambda h}}{p(\lambda)(1+\gamma)} \int_{0}^{a} \sqrt{t} \phi(t) J_{\frac{3}{2}}(\lambda t) d t+\right. \\
\left.\left(e^{\lambda h}\left(1-\frac{1}{p(\lambda)}\right)-\frac{e^{\lambda h}}{p(\lambda)}\right) \int_{0}^{b} \sqrt{t} \psi(t) J_{\frac{1}{2}}(\lambda t) d t\right] J_{1}(\lambda r) d \lambda \\
\tau_{z \theta}^{1}(r, h)=G_{1} \int_{0}^{\infty} \lambda^{\frac{3}{2}} \int_{0}^{b}\left[\sqrt{t} \psi(t) J_{\frac{1}{2}}(\lambda t) d t\right] J_{1}(\lambda r) d \lambda
\end{gathered}
$$

The second part of the integrals in Eq. (29) converge quickly as their limits $r \rightarrow a$ and $r \rightarrow b$ automatically vanishes; however, the limits of the other two integrals analyzed asymptotically as follows. Using the relation $J_{1}(\lambda R)=-\frac{1}{R} \frac{d}{d R} J_{0}(\lambda R)$, we obtain

$$
\begin{gathered}
\tau_{z \theta}^{1}(r, 0)=-G_{1} \int_{0}^{a} \sqrt{t} \phi(t) d t \int_{0}^{\infty} \frac{\lambda^{\frac{1}{2}}}{p(\lambda)(1+\gamma)} J_{\frac{3}{2}}(\lambda t) J_{0}(\lambda r) d \lambda-G_{1} \int_{0}^{\infty} \lambda^{\frac{3}{2}} \frac{e^{-2 \lambda h}}{p(\lambda)(1+\gamma)} \\
\left.\left.\left.\int_{0}^{a} \sqrt{t} \phi(t)\right]_{\frac{3}{2}}(\lambda t) d t+\left(e^{\lambda h}\left(1-\frac{1}{p(\lambda)}\right)-\frac{e^{\lambda \lambda}}{p(\lambda)}\right) \int_{0}^{b} \sqrt{t} \psi(t)\right]_{\frac{1}{2}}(\lambda t) d t\right] J_{1}(\lambda r) d \lambda \\
\tau_{z \theta}^{1}(r, h)=-G_{1} \int_{0}^{b} \sqrt{t} \psi(t) d t \int_{0}^{\infty} \lambda^{\frac{1}{2}} J_{\frac{1}{2}}(\lambda t) J_{0}(\lambda r) d \lambda
\end{gathered}
$$

For large values of $\lambda$, we use the following asymptotic behavior of the Bessel function of the first $J_{\nu}(\lambda) \cong \sqrt{\frac{2}{\lambda \pi}} \cos \left(\lambda-v \frac{\pi}{2}-\frac{\pi}{4}\right)$ and using the following integral formulas for the first infinite integral in the right part of the Eqs. (31) and (32), respectively,

$$
\begin{gathered}
\int_{0}^{\infty} \cos (\lambda t) J_{0}(\lambda r) d \lambda= \begin{cases}\frac{1}{\sqrt{\left(r^{2}-t^{2}\right)}} & t<r \text { and } \\
0 & t>r\end{cases} \\
\int_{0}^{\infty} \sin (\lambda t) J_{0}(\lambda r) d \lambda= \begin{cases}0 & t<r \\
\frac{1}{\sqrt{\left(t^{2}-r^{2}\right)}} & t>r\end{cases}
\end{gathered}
$$

As $\mathrm{p}(\lambda) \rightarrow 1$ as $\lambda \rightarrow \infty$, Eqs. (31) and (32) become

$$
\begin{gathered}
\tau_{z \theta}^{1}(r, 0)=\frac{G_{1}}{\gamma+1} \sqrt{\frac{2}{\pi}} \frac{d}{d r} \int_{0}^{a} \frac{\phi(t)}{\sqrt{\left(r^{2}-t^{2}\right)}} d t+R_{1}(r) \\
\tau_{z \theta}^{1}(r, h)=-G_{1} \sqrt{\frac{2}{\pi}} \frac{d}{d r} \int_{0}^{b} \frac{\psi(t)}{\sqrt{\left(t^{2}-r^{2}\right)}} d t
\end{gathered}
$$

where

$$
\begin{aligned}
R_{1}(r)=-G_{1} \int_{0}^{\infty} \lambda^{\frac{3}{2}} & {\left[\frac{e^{-2 \lambda h}}{p(\lambda)(1+\gamma)} \int_{0}^{a} \sqrt{t} \phi(t) J_{\frac{3}{2}}(\lambda t) d t+\right.} \\
& \left.\left(e^{\lambda h}\left(1-\frac{1}{p(\lambda)}\right)-\frac{e^{\lambda h}}{p(\lambda)}\right) \int_{0}^{b} \sqrt{t} \psi(t) J_{\frac{1}{2}}(\lambda t) d t\right] J_{1}(\lambda r) d \lambda
\end{aligned}
$$

Now integrating by parts, we get

$\tau_{z \theta}^{1}(r, 0)=\frac{G_{1}}{\gamma+1} \sqrt{\frac{2}{\pi}}\left[\frac{a \phi(a)}{r \sqrt{\left(r^{2}-a^{2}\right)}} \int_{0}^{a} \frac{t \phi^{\prime}(t)}{\sqrt{\left(r^{2}-t^{2}\right)}} d t\right]+R_{1}(r)$

We note that the infinite integrals in the preceding expressions are convergent throughout the medium except at the singular points $r \rightarrow a^{+}$, which occupy the crack boundary.

$\tau_{z \theta}^{1}(r, h)=G_{1} \sqrt{\frac{2}{\pi}}\left[\frac{b \psi(b)}{r \sqrt{\left(b^{2}-r^{2}\right)}}-\int_{r}^{b} \frac{t \psi^{\prime}(t)}{\sqrt{\left(r^{2}-t^{2}\right)}} d t\right]$

In this case, the integral in the above relation converges quickly and the integral is bonded as $r \rightarrow b^{-}$. As a result, we obtain a square root singularity at $r=b$ and the constant $\psi(b)$ is the measure of the strength of singularity at the vicinity of the rigid inclusion. By using the following transformations $\phi(a)=\frac{4 a \omega}{\sqrt{2 \pi}} \Phi_{N}, \psi(b)=\frac{4 b \omega}{\sqrt{2 \pi}} \Psi_{N}$.

we obtain the stress intensity factor at the edge of the crack and at the rim of the disc

$$
K_{I I I}^{a}=\frac{-4 G_{1} \omega \sqrt{a}}{(1+\gamma) \sqrt{\pi}} \Phi_{N}
$$




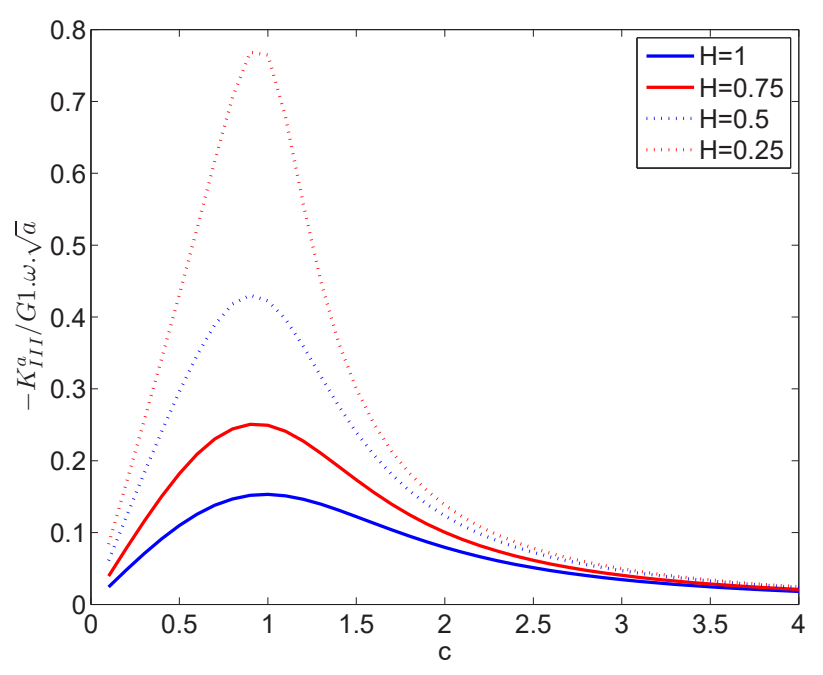

Figure 2: Variation of the normalized stress intensity factor at the edge of the crack $K_{I I I}^{a}$ with a/b.

$$
K_{I I I}^{b}=\frac{4 G_{1} \omega \sqrt{b}}{(1+\gamma) \sqrt{\pi}} \Psi_{N}
$$

Figure 2 shows the variation of the normalized stress intensity factor $K_{I I I}^{a}$ at the edge of the crack defined by Eq. (37a) against $c$ for various values of the layer thickness $H=$ $1,0.75,0.5,0.25$ calculated using the shear modulus ratio $y=1$. It is observed that the values of stress intensity factor increase and attain its maximum values at $c=1$, and with the increase in the value of $c$, the stress intensity factor decreases. In addition, the effect of the axial distance between the crack and the disc $H$ on the stress intensity factor is also shown in this figure. The increase in the distance $H$ induces the decrease in stress intensity factor for all the values of parameter $c$. Figure 3 illustrates the variation of the stress intensity factor at the crack because of variations in shear modulus ratio $y$ for different values: the normalized crack size $c=0.25,0.5,0.75,1$ and the layer thickness $H=1$. We observe from the figure that as the shear parameter $y$ increases, the stress intensity factor decreases for all values of $c$.

Figure 4 illustrates the variation of the normalized stress intensity factor $K_{I I I}^{b}$ at the edge of the rigid inclusion defined by Eq. (37b) versus $c$ for $H=1,0.75,0.5$, and 0.25 . Relatively, small variation for smaller values of $c$ and considerable variation for larger values of $c$ are observed. Also, the interaction between the cracks and the rigid disc is greater when the cracks are closer to the disc $K^{\text {In }}$ ${ }_{K}$ d $G_{\text {ition }}$ to the interaction, the stress intensity factor $K_{I I I}^{\text {II }}$ $K_{I I I}$ increases as the crack radius increases. From the

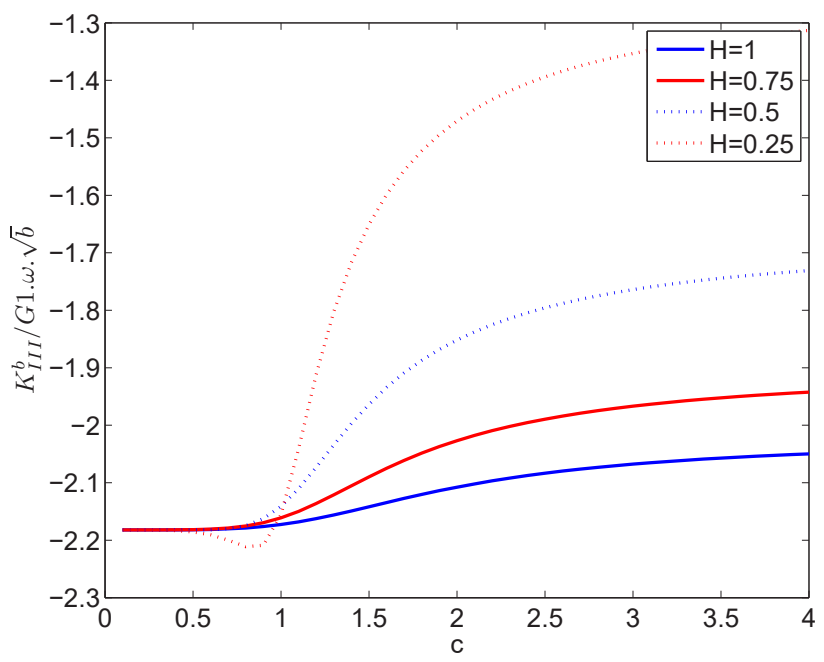

Figure 3: Variation of the normalized stress intensity factor at the edge of the crack $K_{I I I}^{a}$ with $\mathrm{G}_{1} / \mathrm{G}_{2}$.

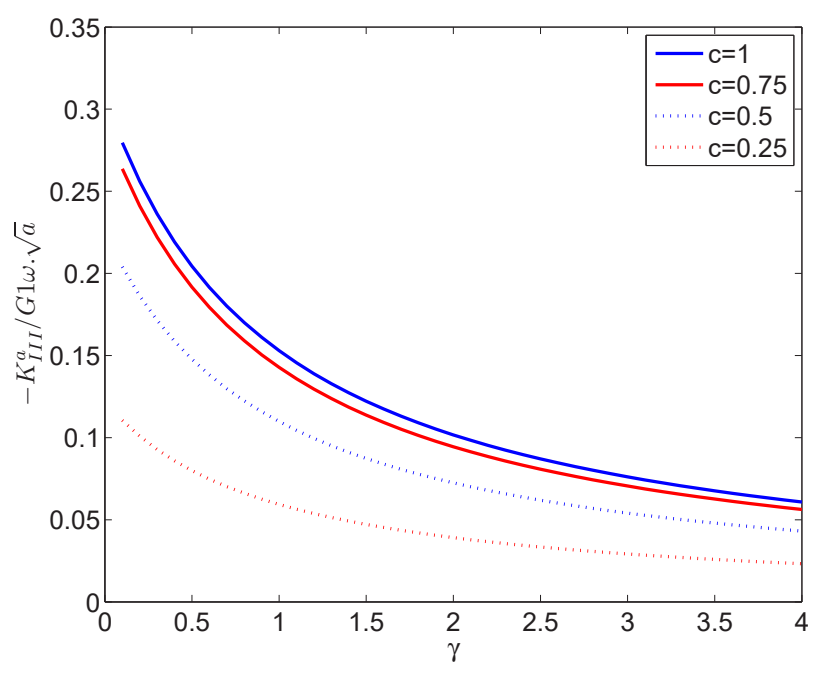

Figure 4: Variation of the normalized stress intensity factor at the edge of the rigid disc $K_{I I I}^{b}$ with a/b.

formulation and the presented figures, conclusions may be deduced:

1. Singularity at the edge of the internal crack and the internal rigid inclusion is observed (The results seem to agree with the previous works (Low [16], Dhawan [17], and Madani and Kebli [20]).

2. The Mode III stress intensity factor $K_{I I I}^{b}$ at the edge of the crack is negative, and it decreases with increasing $y$.

3. There is considerable interaction between the cracks and the rigid inclusion when $a / b$ is large and the cracks are close to the rigid inclusion. 


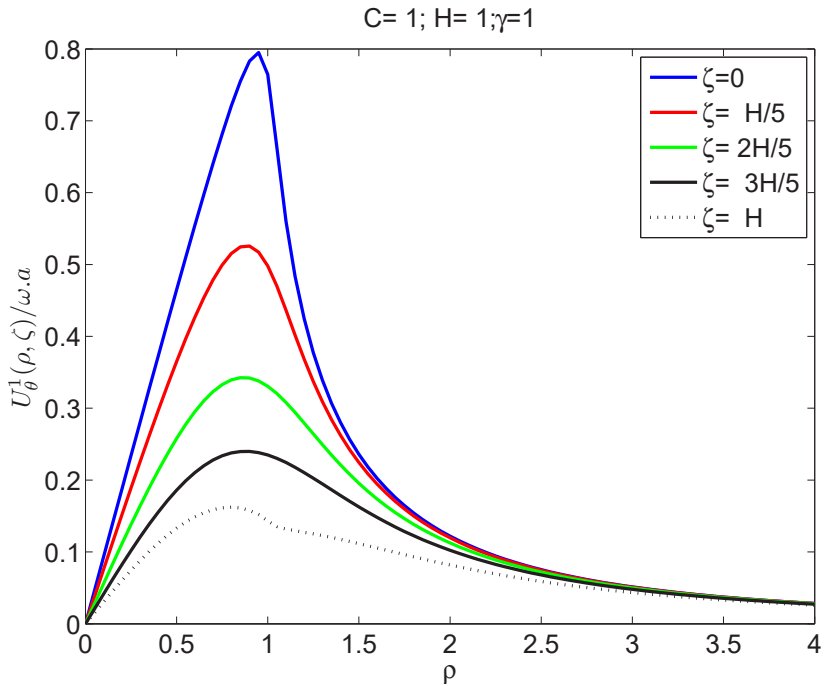

Figure 5: Tangential displacement $u_{\theta}{ }^{1}$ versus $\rho$ for various $\xi, 0 \leq z \leq h$.

\subsection{The moment required to produce rotation of the disc}

The torque required to sustain the rotation of the disc can be computed using the following equation:

$$
T=2 \pi \int_{0}^{b} r^{2} \tau_{z \theta}^{1}(r, 0) d r
$$

Using the relation $\int_{0}^{b} r^{2} J_{1}(\lambda r) d r=\frac{b^{2}}{\lambda} J_{2}(\lambda b)$, we get

$$
T=2 \pi b^{2} G_{1} \int_{0}^{\infty}\left[A_{1}(\lambda)+B_{1}(\lambda)\right] J_{2}(\lambda b) d \lambda
$$

Here, as the moment is applied only to the rigid inclusion, the integrand is expressed in terms of $\psi(t)$. Substituting the values of $A 1(\lambda)$ and $B 1(\lambda)$ from Eqs. (12) and (13) into Eq. (39) and using the asymptotic behavior of the Bessel function of the first kind $J_{\frac{1}{2}}$, we find that

$$
T=2 \sqrt{2} \pi b^{2} G_{1} \int_{0}^{b} \psi(t) d t \int_{0}^{\infty} \sin (\lambda t) J_{2}(\lambda b) d \lambda
$$

Taking into account the relation $\int_{0}^{\infty} \sin (\lambda t) J_{2}(\lambda b) d \lambda=\frac{2 t}{b^{2}}$, we obtain the moment applied to the inclusion

$$
T=4 \sqrt{2 \pi} G_{1} \int_{0}^{b} \psi(t) d t
$$

By using the following transformations $t=b u$ and $\psi(b u)=\frac{4 b \omega}{\sqrt{2 \pi}} \Psi_{\mathbf{u}}$.

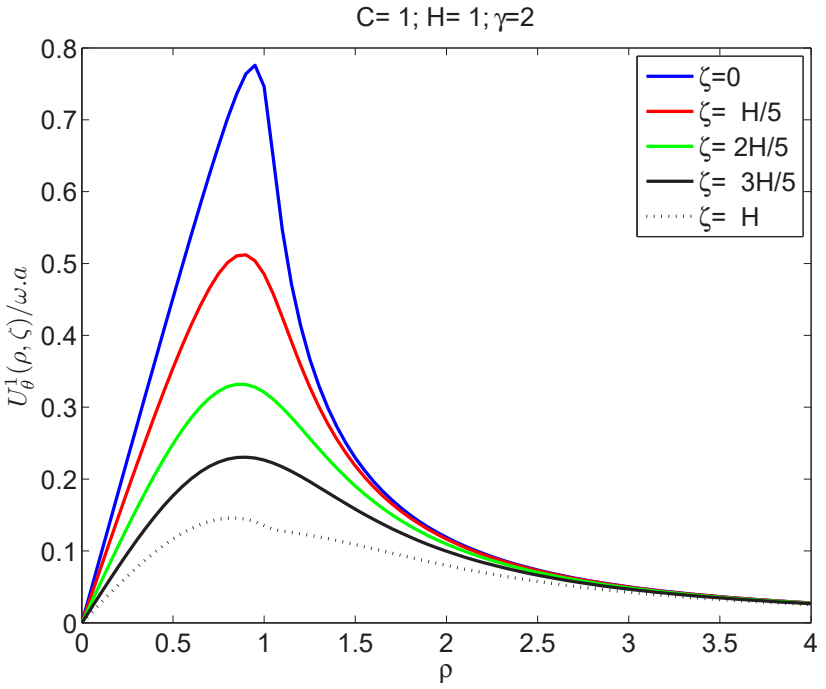

$$
T=16 \omega b^{3} G_{1} \int_{0}^{b} u \Psi(u) d u
$$

The moment required to effect the rotation $\omega$, when the medium contain no crack, can be formulated as $T_{0}=\frac{16 \omega b^{3} G_{1}}{3}$. Equation 42 can be expressed as

$$
\frac{T}{T_{0}}=3 \int_{0}^{b} u \Psi(u) d u
$$

The problem of inclusion-crack interaction has a remarkable use in the design of composite anchoring systems with flat disc inclusions, in deep foundations in a geological medium, in in situ load tests at the base of a borehole, in injection anchoring regions in soft rock masses with sealing materials, or when penetrating single-propelled anchors in steep soil masses, such as over-consolidated clays.

\subsection{Displacement and stress fields}

The results for the variation of the normalized displacement $u^{(i)}(\rho, \xi) / \omega a$ and stress $\tau^{(i)}(\rho, \xi) / G_{i} \omega a$ with $\rho=r / b$ are shown graphically in Figures 5-8 for the different values of the dimensionless axial distances $\xi=z / b$. For each region, five different axial distances are selected as $I(\xi=0 ; H / 5 ; 2 H / 5$; $3 H / 5 ; H)$ and $I I(\xi=H ; 6 H / 5 ; 7 H / 5 ; 8 H / 5 ; 2 H)$, with the particular values of the height $H=1$, the dimensionless 

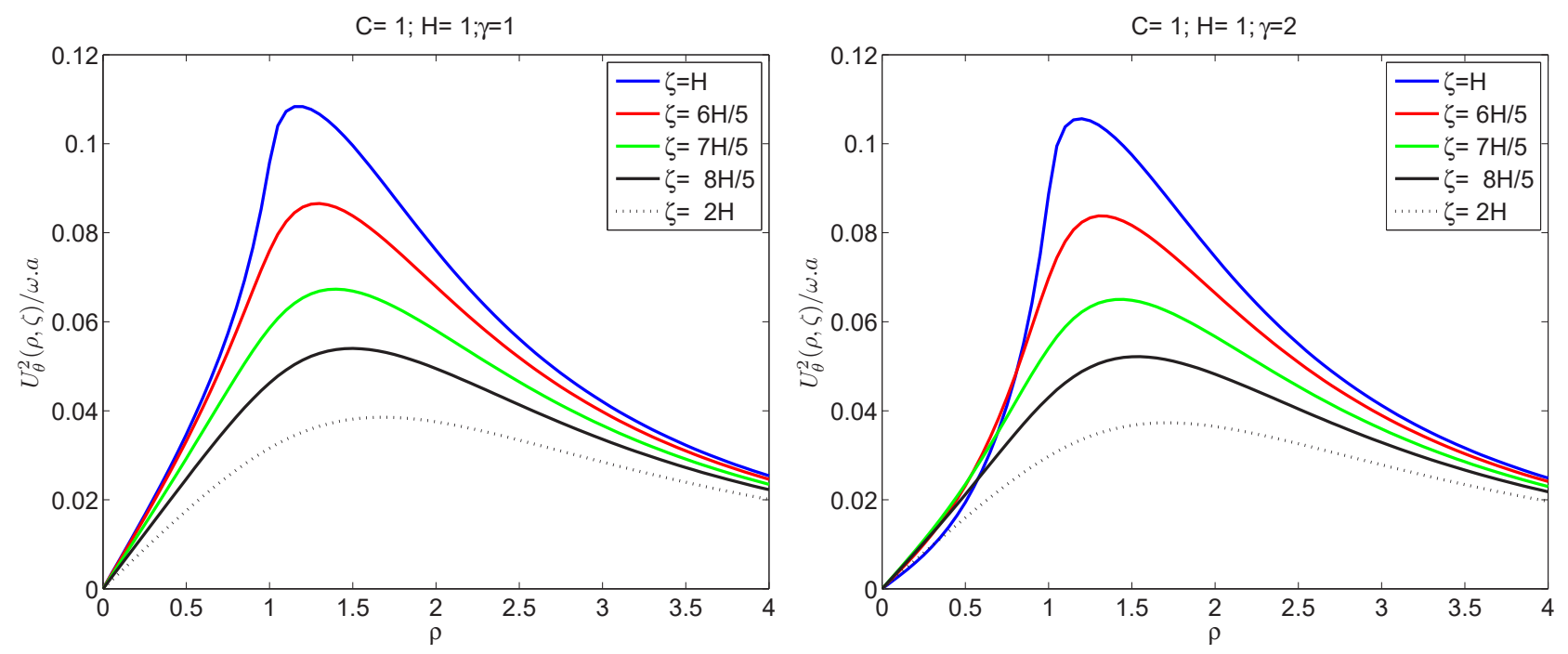

Figure 6: Tangential displacement $u_{\theta}^{2}$ versus $\rho$ for various $\xi, z \geq h$.
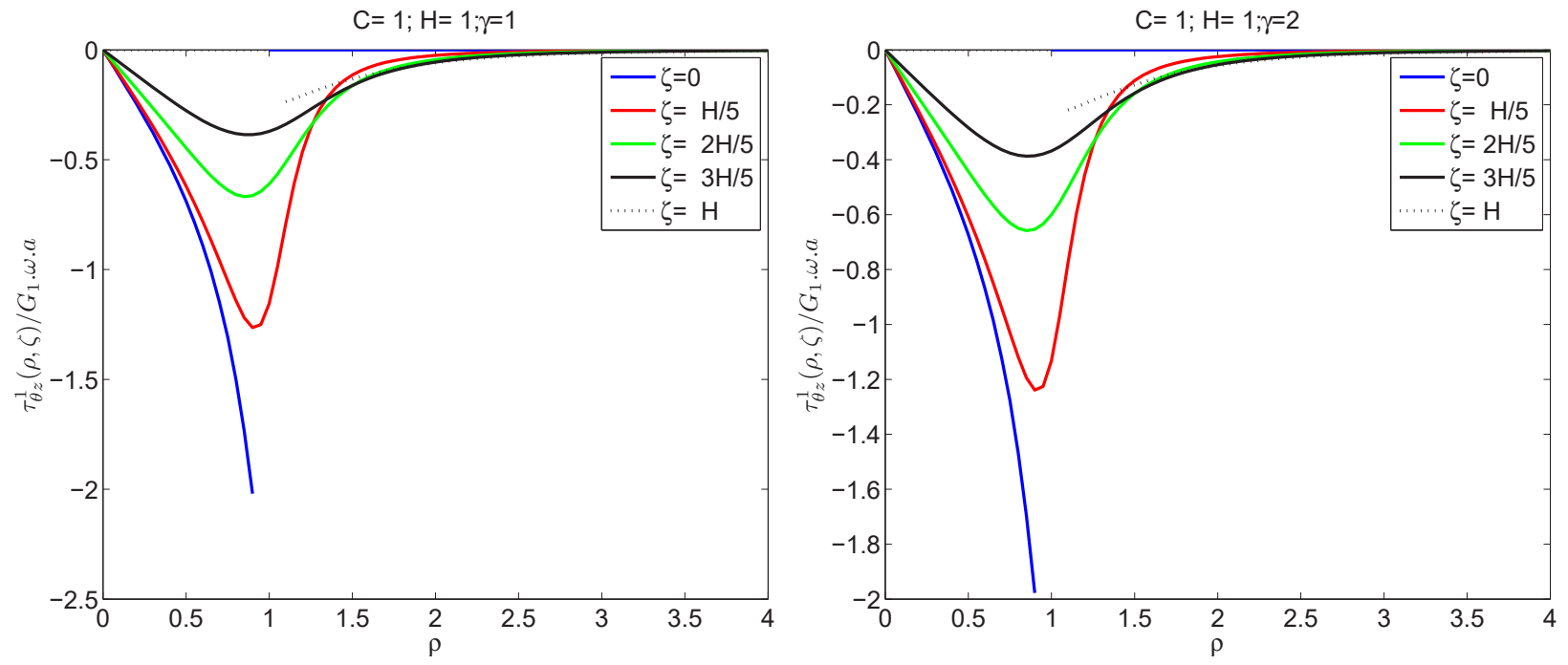

Figure 7: Shear stress $\tau_{\theta z}{ }^{1}$ versus $\rho$ for various $\xi, 0 \leq z \leq h$.

crack size $c=1$ and the shear parameter $y=1$ and $y=2$. The variation of the normalized displacements is shown in Figures 5 and 6. We notice that the displacements in the two regions increase at first, reach maximum values at $\rho=$ $c$, and then decrease out of the disc band with increasing $\rho$. The distribution of the shear stresses in the elastic medium are also discussed and shown in Figures 7 and 8. The stresses initially increase, attain its maximum values, and then with the increase in the value of $\rho$, the stresses go on decreasing.

\section{Conclusion}

In this article, an axisymmetric torsion of a rigid disc embedded in the interior of a homogeneous elastic layer sandwiched between two half-spaces containing two interface cracks is analytically addressed. By using the Hankel integral transformation and its inverse, the mixed boundary value problem is reduced to a system of dual integral equations, which are reduced to a Fredholm integral equation system of the second kind. The numerically computed results of the displacements, the stresses, and the stress intensity factors are presented 


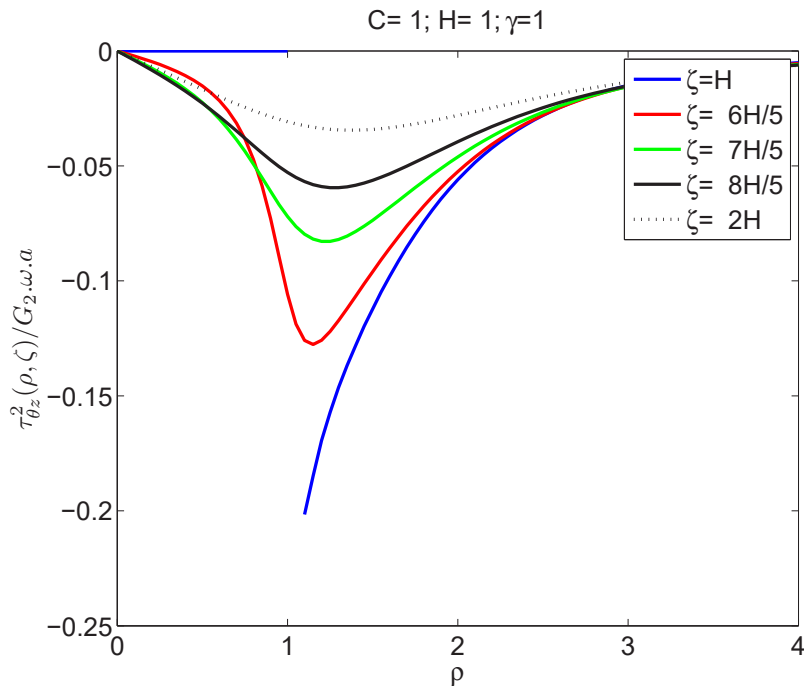

Figure 8: Shear stress $\tau_{\theta z}{ }^{2}$ versus $\rho$ for various $\xi, z \geq h$.

graphically for some dimensionless parameters. The numerical results show that the discontinuities around the crack and the inclusion cause a large increase in the stresses that decay with distance from the disc loaded. Furthermore, the dependence of the stress intensity factor on the disc size, the distance between the crack and the disc and the nonhomogeneity parameter is observed.

\section{References}

[1] Menshykov, O.V., Menshykov, V.A. and Guz, I.A.: The contact problem for an open penny-shaped crack under normally incident tension-compression wave. Eng. Fract. Mech. 75(5), 1114-1126 (2008).

[2] Selvadurai, A.P.S.: Asymmetric displacements of a rigid disc inclusion embedded in a transversely isotropic elastic medium of infinite extent . Int. J. Sci. 18, 979-686 (1980)

[3] Selvadurai, A.P.S.: Rotary oscillations of a rigid disc inclusion embedded in an isotropic elastic infinite space. Int. J. Solids. Struct. 17, 493-498 (1981)

[4] Reissner E., Sagoci H.F.: Forced torsion oscillation of an halfspace I, Int. J. Appl. Phys., 15, (1944), 652-654

[5] Sneddon I.N.: Note on a boundary value problem of Reissner and Sagoci, Int. J. Appl. Phys., 18, (1947), 130-132

[6] Collins W.D.: The forced torsional oscillations of an elastic halfspace and an elastic stratum, Pro. London. Math. Society, 12, (1962), 226-244

[7] Gladwell G.M.L.: The forced torsional vibration of an elastic stratum, Int. J. Eng. Sci., 7, (1969), 1011-1024

[8] Pak R.Y.S., Saphores J.D.M.: Torsion of a rigid disc in a halfspace, Int. J. Eng Sci., 29, (1991),

[9] $1-12$

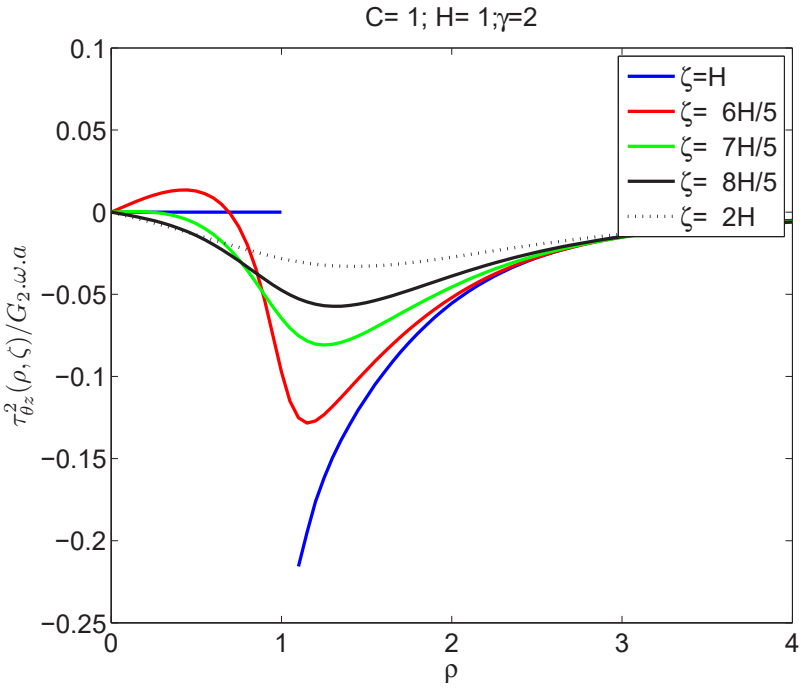

[10] Bacci A., Bennati, S.: An approximate explicit solution for the local torsion of an elastic layer, Mech. Struct. Mach., 24, (1996), 21-38

[11] Singh B.M., Danyluk H.T., Vrbik J., Rokne J., Dhaliwal R.S.: The Reissner-Sagoci Problem for a Non-homogeneous Half-space with a Surface Constraint, Meccanica, 38, (2003), 453-465

[12] Guo-cai W., Long-zhu C.J.: Torsional oscillations of a rigid disc bonded to multilayered poroelastic medium, Int. Zheijang. Univ. Sci., 6(3), (2005), 213-221

[13] Yu H.Y.: Forced torsional oscillations of multilayered solids, Int. J. Eng. Sci., 46, (2008), 250-259

[14] Pal P.C., Mandal D., Sen B.: Torsional Oscillations of a Rigid Disc Embedded in a Transversely Isotropic Elastic Half-Space, Adv. Theor. Appl. Mech., 4, (2011), 177-188

[15] Ahmadi S.F.; Eskandari M.: Rocking rotation of a rigid disk embedded in a transversely isotropic half-space, Civil Eng. Infra. J., 47, (2014), 125-138

[16] Sih G.C., Chen E.P.: Torsion of a laminar composite debonded over a penny-shaped area, J. Franklin Inst., 293, (1972), 251-261

[17] Low R. D.: On the torsion of elastic half space with embedded penny-shaped flaws, J. Appl. Mech., 39, (1972), 786-790

[18] Dhawan G. K.: On the torsion of elastic half-space with pennyshaped crack, Defense. Sci. J., 24, (1974), 15-22

[19] Basu S.; Mandal S.C.: Impact of Torsional Load on a PennyShaped Crack in an Elastic Layer Sandwiched Between Two Elastic Half-Space, Int. J. Appl. Comput. Math , 2, (2016), 533-543

[20] Debnath, L., Bhatta, D.:, Integral transforms and their applications, Chapman Hall, CRC, (2007) Madani, F., Kebli, B.: Axisymmetric Torsion of an Internally Cracked Elastic Medium by Two Embedded Rigid Discs, Mechanics and Mechanical Engineering , 21, (2017), 363-377

[21] Kythe, P. K.; Puri, P.: Computational methods for linear integral equations. Birkhäuser, Boston (2002)

[22] Atkinson, K.: The numerical solution of integral equations of the second kind. Cambridge, University Press. New York (1997) 